\title{
Scavenger receptor-mediated endocytosis facilitates RNA interference in the desert locust, Schistocerca gregaria
}

\author{
N. Wynant, D. Santos, P. Van Wielendaele and \\ J. Vanden Broeck \\ Molecular Developmental Physiology and Signal \\ Transduction, Department of Animal Physiology and \\ Neurobiology, KU Leuven, Leuven, Belgium
}

\begin{abstract}
RNA interference (RNAi) has become a widely used loss-of-function tool in eukaryotes; however, the delivery of double-stranded (ds)RNA) to the target cells remains a major challenge when exploiting the RNAi-technology. In insects, the efficiency of RNAi is highly species-dependent. Yet, the mechanism of cell entry in insects has only been characterized in a cell line of the fruit fly, Drosophila melanogaster, a species that is well known to be poorly amenable to environmental RNAi. In the present paper, we demonstrate that silencing vacuolar H-ATPase 16 (vha16) and clathrin heavy chain (clath), two components of the Clathrin-dependent endocytosis pathway, together with pharmacological inhibition of scavenger receptors with polyinosine and dextran sulphate, can significantly attenuate the highly robust RNAi response in the desert locust, Schistocerca gregaria.
\end{abstract}

Keywords: RNAi, double stranded RNA (dsRNA), insect, sid1, pattern recognition, environmental, systemic.

\section{Introduction}

RNA interference (RNAi) is a mechanism of sequencespecific gene regulation triggered by double-stranded

Correspondence: Niels Wynant, Molecular Developmental Physiology and Signal Transduction, Department of Animal Physiology and Neurobiology, KU Leuven, Naamsestraat 59, P.O. Box 02465, B-3000 Leuven, Belgium. Tel.: +32 16323900; fax: +32 16323902; e-mail: niels.wynant@ bio.kuleuven.be
(ds)RNA (Fire et al., 1998). While RNAi is known to be involved in antiviral immunity (Waterhouse et al., 2001; Zambon et al., 2006), genome maintenance (Lippman \& Martienssen, 2004) and regulation of endogenous gene expression (Hutvagner \& Zamore, 2002), it has also become a widely used tool to knock down and analyse the function of genes in eukaryotes (Hardy et al., 2010). In addition, several recent studies have shown that RNAi may also contribute to strategies for selectively controlling agricultural pests, including a number of insect species (Baum et al., 2007; Mao et al., 2007; Huvenne \& Smagghe, 2010); however, a major challenge in exploiting the RNAi technology remains the introduction of dsRNA into the cells (Perrimon et al., 2010; Yu et al., 2013).

Many organisms display RNAi after injection or oral delivery of dsRNA. This phenomenon, in which RNAi is established in tissues distant from the site of dsRNA administration, is referred to as environmental (env)RNAi. This type of RNAi requires the cellular uptake of dsRNA from the extracellular environment, but not necessarily the spreading of the RNAi-signal from cell to cell (Whangbo \& Hunter, 2008). Following a screen with mutant Caenorhabditis elegans worms, the transmembrane channel protein Systemic RNA Interference Deficient-1 (SID1) was identified as a key player in the cellular uptake of dsRNA (Winston et al., 2002; Duxbury et al., 2005). Expression of the Ce-sid1 sequence in Drosophila Schneider (S)2 cells, which lack a sid1 homologous sequence, significantly enhanced the envRNAi response (Feinberg \& Hunter, 2003). In addition, homologous sequences of sid1 in fish and mammals have been shown to mediate dsRNA import (Duxbury et al., 2005; Ren et al., 2011; Elhassan et al., 2012). The genomes of most insects, with the exception of dipterans, also possess genes related to Ce-sid1. Yet, their definite functional role in RNAi remains unclear. For example, Tribolium castaneum and Locusta migratoria display a robust envRNAi response, but their sid1 like sequences do not seem to mediate uptake of dsRNA (Bucher et al., 2002; Tomoyasu et al., 2008; Luo et al., 
2012). By contrast, in Apis mellifera, the involvement of sid1 like in the envRNAi response was suggested, since administration of dsRNA induced an up-regulation of the Am-sid1 like transcript levels (Aronstein et al., 2006).

The best-studied insect model, Drosophila melanogaster, as well as many lepidopterans are poorly sensitive towards envRNAi, since injection of dsRNA into the body cavity is not an effective method to induce RNAi (Miller et al., 2008; Belles, 2010; Terenius et al., 2011). For example, in the larval stage of D. melanogaster, the haemocytes (and the haemocyte-like S2 cell line) seem to be the only cell type that can take up dsRNA (Miller et al., 2008). Yet, since sid1 like sequences are absent in the genomes of these flies, S2 cells must use alternative dsRNA-uptake mechanisms. Two independent functional screens demonstrated that dsRNA enters S2 cells via scavenger receptor (SR)-mediated endocytosis (Saleh et al., 2006; Ulvila et al., 2006). Saleh et al. (2006) suggested that Vacuolar H-ATPase 16 (Vha16) plays an important role in the redirection of exogenous dsRNA from the vesicular location of the standard endocytotic uptake route into the cytoplasm, where the RNAi-machinery is localized. In contrast to D. melanogaster (Belles, 2010; Miller et al., 2012), the desert locust, Schistocerca gregaria, displays a very robust envRNAi response, as virtually all tissues are sensitive towards injection of dsRNA in the body cavity and only small quantities of dsRNA are needed (Wynant et al., 2012). These characteristics make this species amenable to the study of the mechanisms of envRNAi in insects.

In the present study, we demonstrate that two components of Clathrin-dependent endocytosis, namely vha16 and clathrin heavy chain (clath), and SRs play an important role in the in vivo envRNAi response of $S$. gregaria, while loss-of-function experiments and in silico analyses suggest that $S g$-SID1 like plays no major role in the uptake of dsRNA.

\section{Results}

\section{Silencing components involved in Clathrin-dependent endocytosis}

To assess the involvement of Clathrin-dependent endocytosis in the envRNAi response in S. gregaria, we silenced clath and vha16 that mediate formation of coated vesicles and lysosomal acidification, respectively. After injection of $150 \mathrm{ng}$ of the corresponding dsRNA into the abdominal body cavity (hemocoel), silencing of these two genes in the midgut was confirmed with quantitative PCR (qPCR) by comparing the transcript levels with a control group that was injected with green fluorescent protein (gfp) dsRNA (Fig. 1). The knockdown of vha16 was assessed 6 days after the dsRNA injection; however, silencing of clath gene expression induced death of the
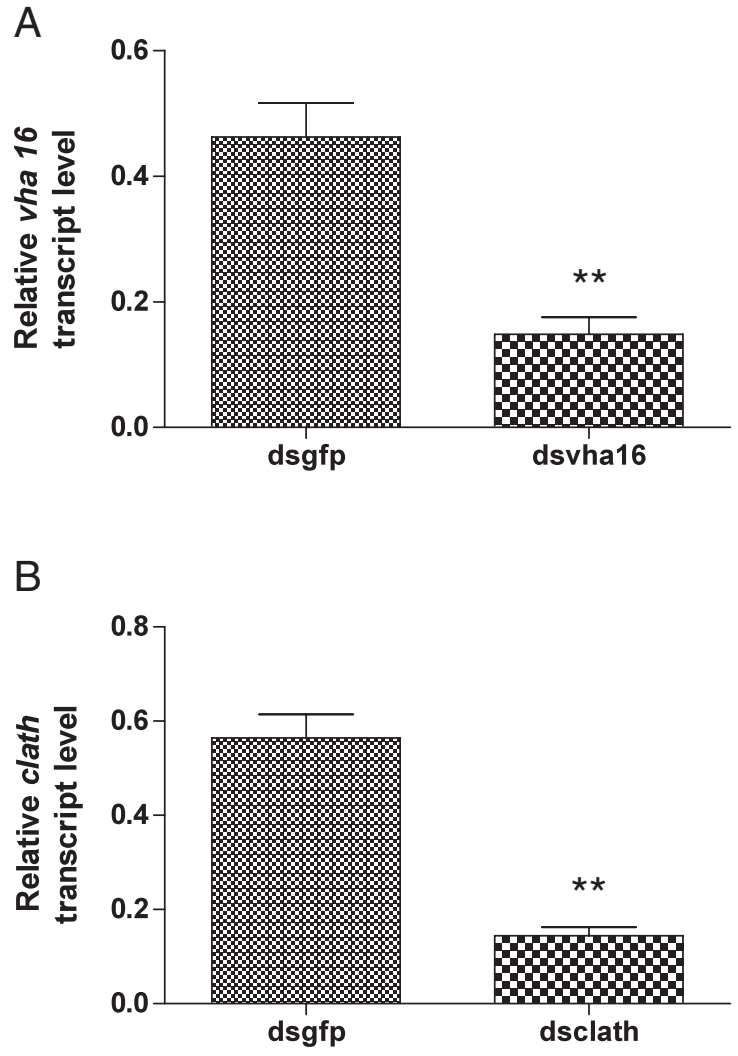

Figure 1. Gene silencing of $(A)$ vha16 and $(B)$ clath was verified by measuring transcript levels in the midgut. The knockdown was determined 6 days after injection of $150 \mathrm{ng}$ of double-stranded (ds)RNA of vha16 and 4 days after injection of $150 \mathrm{ng}$ of clath dsRNA. The transcript levels were compared with locusts injected with $150 \mathrm{ng}$ of dsRNA of $g f p\left({ }^{* *}: P<0.005 ; n \geq 6\right)$.

injected locusts, starting from 7 days after the dsRNA administration; therefore, all experiments with clath dsRNA were performed 4 days after the injection. At that time, there were not yet detectable defects in the behaviour of the locusts.

\section{Endocytosis-based uptake of the RNAi-signal}

An 'RNAi on RNAi' approach was followed to investigate whether these endocytotic pathway components contribute to the envRNAi response in the RNAi-sensitive midgut tissue. First, we silenced vha16 by injection of $150 \mathrm{ng}$ of its transcript-specific dsRNA. Six days later, a second injection was performed with $150 \mathrm{ng}$ alpha-tubulin 1a (tubu) dsRNA. The potency of the tubu knockdown was used as a marker for the envRNAi potency, $16 \mathrm{~h}$ after injection of dstubu. To promote knockdown on the functional protein level, we waited 6 days after the dsvha16 injection, while the knockdown of tubu was assessed $16 \mathrm{~h}$ post injection, since at this early time point of the RNAi response the knockdown was already robust. 
A
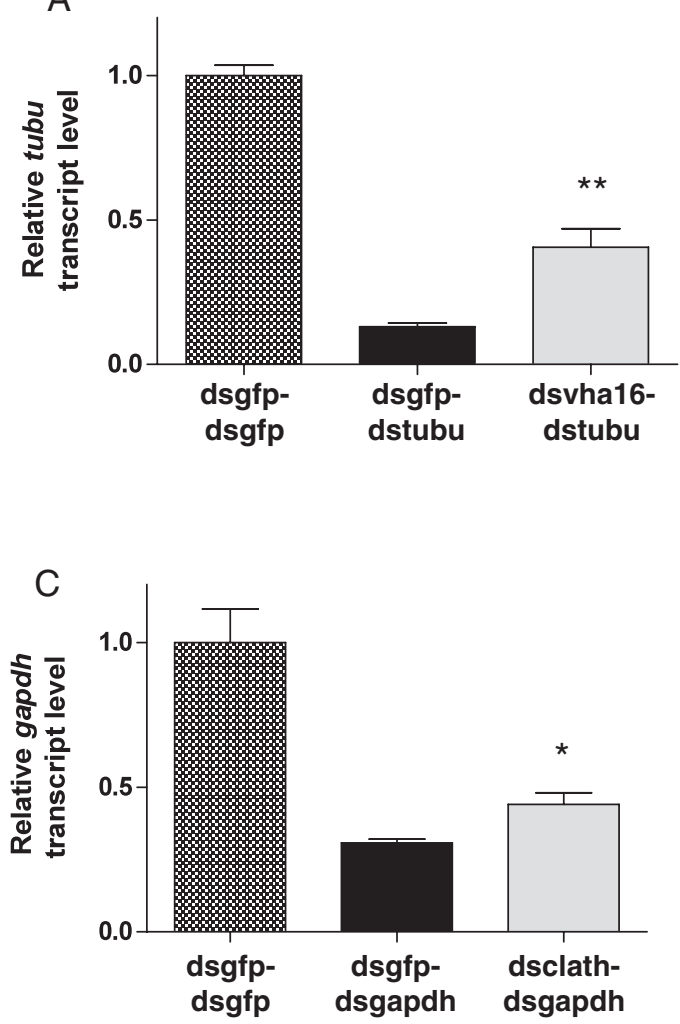

B

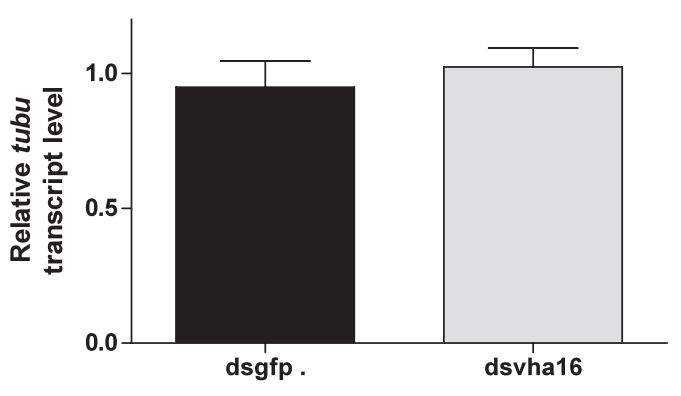

D

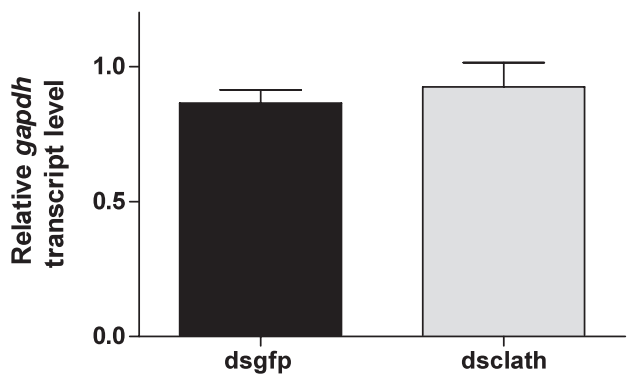

Figure 2. clath and vha16 are involved in mediating the environmental RNA interference response. (A) The locusts were first injected with 150 ng of double-stranded (ds)gfp or dsvha16 and 6 days later with $150 \mathrm{ng}$ of $d s g f p$ or dstubu. The different treatment groups are indicated as dsgfp-dsgfp, dsgfp-dstubu and dsvha16-dstubu. Sixteen hours after the second injection, the tubu transcript level in the midgut was determined by means of qPCR. Statistical analyses were performed between dsgfp-dstubu and dsvha16-dstubu (**: $P<0.005 ; n \geq 8$ ). (B) The tubu transcript levels were determined 6 days after injection of dsgfp or dstubu $(n \geq 6)$. (C) The locusts were first injected with $150 \mathrm{ng}$ of dsgfp or dsclath and 4 days later injected with $150 \mathrm{ng}$ of dsgfp or dsgapdh, indicated as dsgfp-dsgfp, dsgfp-dsgapdh and dsclath-dsgapdh, respectively. Statistical analyses were performed between dsgfp-dsgapdh and dsclath-dsgapdh ( ${ }^{*}: P<0.05 ; n \geq 8$ ). (D) The gapdh transcript level was determined 4 days after injection of dsgfp or dsclath ( $\geq 6$ ).

In addition, the RNAi response was assessed in midgut tissues, as this tissue was previously shown to be highly sensitive towards envRNAi (Wynant et al., 2012). As a control experiment, locusts were injected with dsRNA for gfp and, 6 days later, they were injected with tubu dsRNA. A second control group that was treated twice with gfp dsRNA was also taken into account. Because in this group the tubu gene expression was not silenced, these locusts were used to determine the normal physiological tubu expression levels. Our results show that knocking down the tubu transcript level was significantly less potent when vha16 was down-regulated, i.e. in comparison with the first control group (Fig. 2A). Still, it remained possible that silencing vha16 had some effect on the tubu transcript levels, rather than impairing the uptake of RNAi; therefore, we also silenced vha16 and measured the tubu transcript levels. The tubu expression levels remained indistinguishable upon silencing vha16 from these in the gfp dsRNA-injected control conditions (Fig. 2B). Second, we assessed whether silencing clath could also impair the envRNAi response. To ascertain that the observations were not specific for silencing tubu, we chose a different marker gene, namely glyceraldehyde 3-phosphate dehydrogenese (gapdh), to determine the knockdown potency. The knockdown of gapdh was significantly less robust when clath was down-regulated (Fig. $2 \mathrm{C}$ ), while silencing clath had no direct effect on the gapdh transcript levels (Fig. 2D).

\section{Scavenger receptors are important for envRNAi}

Since no sequence information of $S$. gregaria SRs was available in the $S$. gregaria expressed sequence tag database, we used well-known inhibitors of the SR family, namely polyinosine $[p o l y(I)]$ and dextran sulphate (DS) (Abrams et al., 1992; Pearson et al., 1993, 1995; Krieger \& Herz, 1994; Whitman et al., 2000). Regarding the fact that an adult desert locust possesses, on average, $200 \mu \mathrm{l}$ haemolymph (Lee, 1961), and that administration of $400 \mu \mathrm{g} / \mathrm{ml}$ of poly(I) or DS to the medium of Drosophila L2 cells could inhibit $>85 \%$ of the SR activity (Abrams et al., 1992), $0.1 \mathrm{mg}$ of the inhibitors was injected into the body 
A

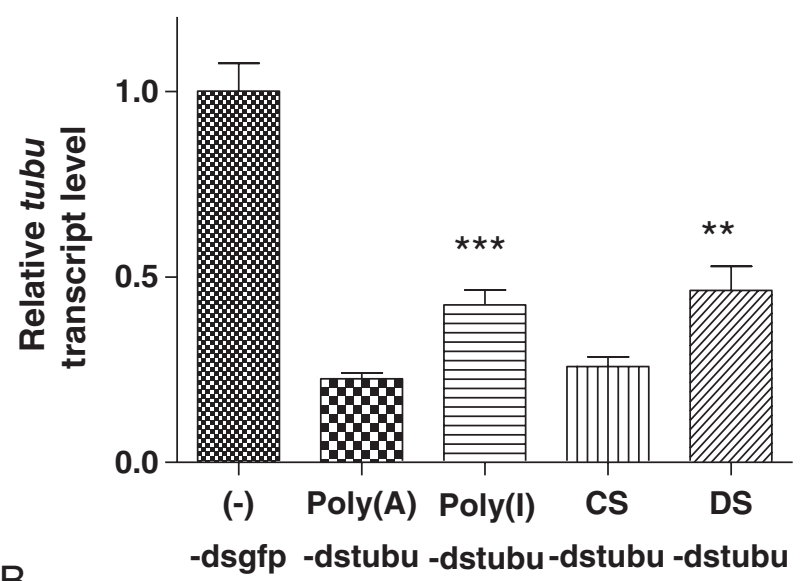

B

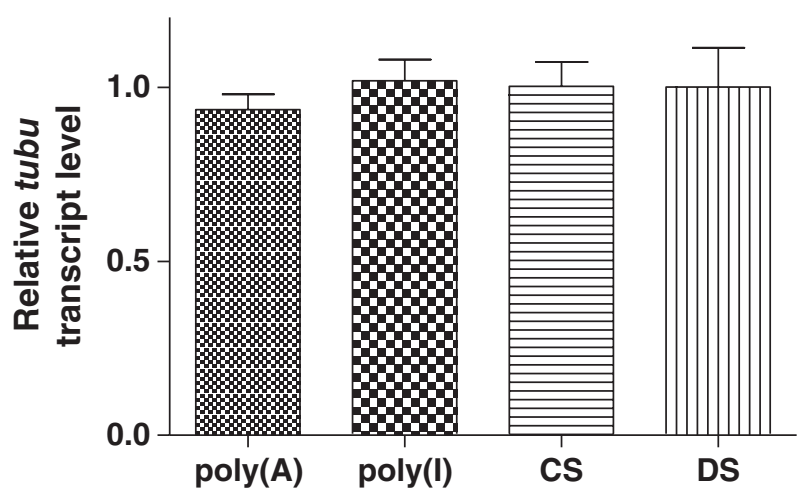

Figure 3. Scavenger receptors facilitate environmental RNA interference envRNAi. (A) The locusts were first injected with $0.1 \mathrm{mg}$ polyinosine (poly[I]), polyadenosine (poly[A]), dextran sulphate (DS) or chondroitin sulphate (CS) and $1 \mathrm{~h}$ later injected with $150 \mathrm{ng}$ of dsRNA of tubu, indicated as poly(I)-dstubu, poly(A)-dstubu, DS-dstubu and CS-dstubu, respectively. A second control group that was once injected with $150 \mathrm{ng}$ of dsgfp, indicated as (-)-dsgfp, was also accounted for. Sixteen hours after the dsRNA injection, the tubu transcript level in the midgut was determined using quantitative PCR. Statistical analyses were performed between the poly(I)-dstubu and poly(A)-dstubu, and between the DS-dstubu and CS-dstubu groups ( ${ }^{\star \star}: P<0.005 ;{ }^{\star \star \star}: P<0.0001 ; n \geq 16$ ). (B) Injection of poly(I) or DS, in comparison with injection of poly(A) or $\mathrm{CS}$, respectively, had no direct effect on the tubu expression level. The expression levels were determined $1 \mathrm{~h}$ after the injection $(n \geq 6)$.

cavity of the desert locusts (which would correspond to $500 \mu \mathrm{g} / \mathrm{ml}$ in the haemolymph). After $1 \mathrm{~h}, 150 \mathrm{ng}$ of tubu dsRNA was injected and the potency of the tubu knockdown was compared with the control groups, which were injected with the same amount of chemically related substances that do not bind to SRs, polyadenosine [poly $(A)]$ and chondroitin sulphate (CS) (Pearson et al., 1993). Our data showed that blocking SRs significantly inhibited environmental silencing of tubu (Fig. 3A), while similar tubu expression levels were observed after injection of the inhibitors and the control compounds (Fig. 3B).
Silencing sid1 like did not generate detectable effects on the RNAi efficiency

Only a small part of the Sg-sid1 like transcript sequence was available (Wynant et al., 2012). The complete transcript was amplified using rapid amplification of cDNA ends (RACE), which resulted in a sequence with an open reading frame (ORF) of $2364 \mathrm{bp}$. The deduced protein sequence was predicted to have a topological structure that was highly similar to SID1 of $C$. elegans, both containing a signal peptide that is followed by a long $\mathrm{N}$-terminus and 11 transmembrane regions (Fig. 4). Yet, silencing $\mathrm{Sg}$-sid1 like did not generate a clear effect on the RNAi efficiency (Fig. 5B). Nonetheless, the knockdown of sid1 like was verified by means of qPCR (Fig 5A). Small differences were, however, reported upon silencing tubu. Yet, silencing $S g$-sid1 like generated an increase in the physiological tubu transcript levels (Supplementary data, Fig. S1). Since differences in the knockdown of gapdh were not observed, the increase in physiological tubu levels might be a good explanation for the observed differences in the remaining tubu transcript levels. Moreover, sequence comparison of the deduced amino acid sequence of $S g$-sid1 like showed higher sequence similarity with ChUP1 (previously known as TAG-130) than with SID1 of $C$. elegans. Ce-ChUP-1 was also predicted to have a highly similar topological structure to the $S g$-SID1 like protein (Supplementary data, Fig. S2) and comparison of their $\mathrm{N}$-terminal extracellular domain revealed regions with high conservation with $\mathrm{Ce}$-ChUP1, but not with Ce-SID1 (Fig. 5C).

\section{Discussion}

By silencing two distinct components of the Clathrindependent endocytosis machinery and by pharmacological inhibition of SRs, we have demonstrated that SR-mediated uptake contributes to the robust RNAi response in the desert locust, $S$. gregaria. In contrast, knocking down Sg-sid1 like generated no clear effects on RNAi efficiency. Moreover, sequence comparison revealed higher sequence similarity with $\mathrm{Ce}$-ChUP1 than with Ce-SID1. Yet, a study by Tomoyasu et al. (2008) has demonstrated that $\mathrm{Ce}$-ChUP1 is not involved in dsRNA uptake in $C$. elegans, but it mediates the uptake of dietary cholesterol (Valdes et al., 2012). Whether Sg-sid1 like is also involved in cholesterol transport rather than dsRNA uptake remains to be determined, but it should be noted that the effect of $S g$-sid1 like was not assessed at the functional protein level so it remains possible that the protein knockdown was too modest to generate clear phenotypic effects. Sid1 like sequences have been identified in many other insect species. Since these sequences also display higher sequence similarity with $\mathrm{Ce}$-ChUP1, it 

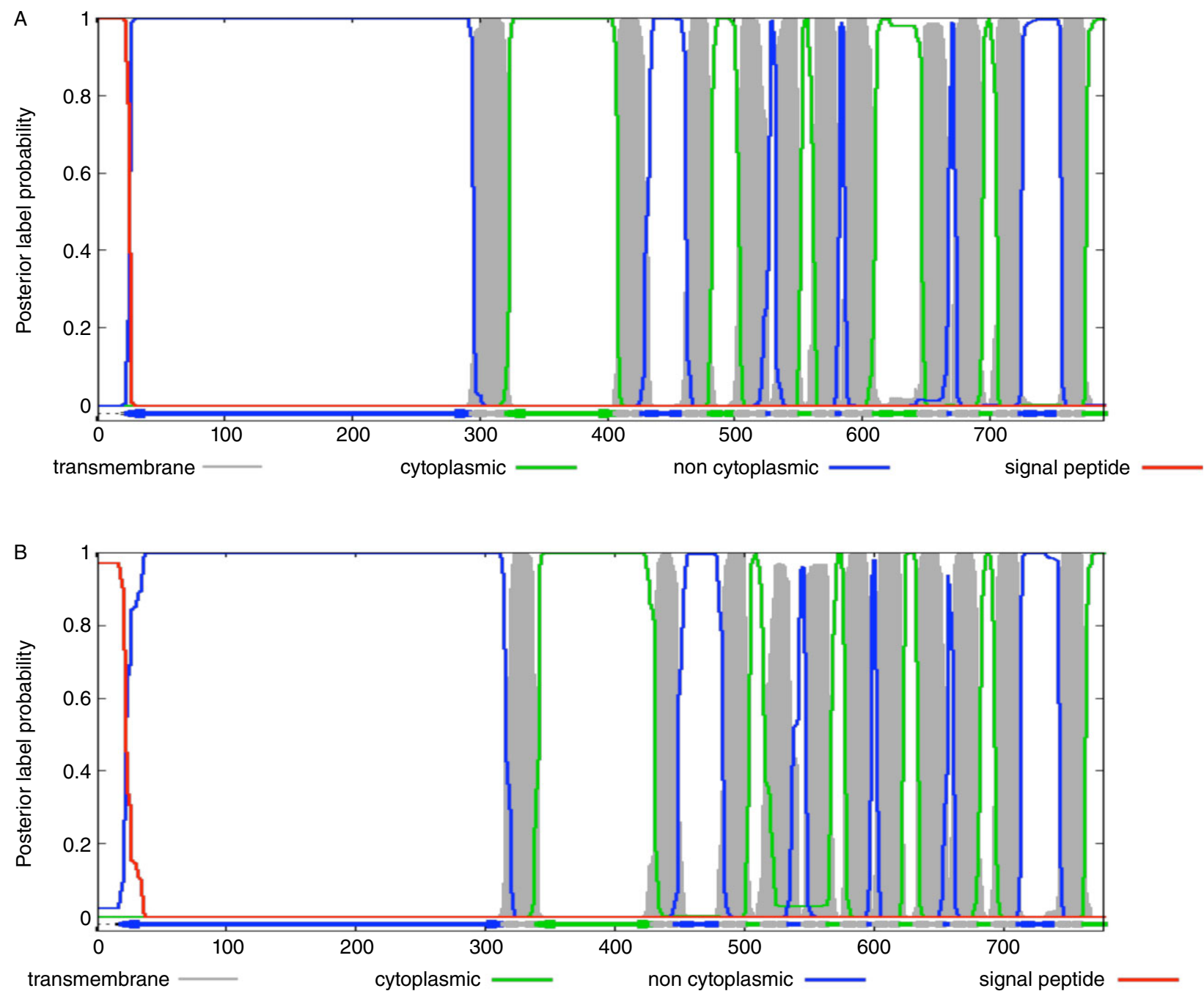

Figure 4. The predicted topologic structure of (A) Sg-SID1 like and (B) Ce-SID1 using PHOBIUs software.

appears to be a misconception that these sequences are true homologues of Ce-SID1.

Although $S$. gregaria and $C$. elegans display a robust envRNAi response, these data suggest on the one hand that $S$. gregaria uses different dsRNA uptake mechanisms. On the other hand, it might seem surprising that S2 cells, an envRNAi-sensitive cell line derived from the largely envRNAi-insensitive $D$. melanogaster, use the same dsRNA entry route (Saleh et al., 2006; Ulvila et al., 2006); however, the tissue specificity and expression levels of components involved in this uptake mechanism may determine the difference in success of the dsRNA transport. As pointed out by several recent reports, the efficiency of the cell-autonomous RNAi machinery may also be a determinate factor for the potency of the knockdown (Swevers et al., 2011; Garbutt \& Reynolds, 2012). The observation that the envRNAi response was only partially inhibited may still suggest the presence of alter- native parallel dsRNA-uptake mechanisms. Nevertheless, we have previously demonstrated that injection of pg amounts of dsRNA per mg tissue can already evoke a clearly detectable RNAi knockdown in $S$. gregaria (Wynant et al., 2012), therefore, one can expect that residual cell entry of dsRNA, attributable to incomplete silencing or inhibition of the uptake mechanisms, would still result in activation of the RNAi response. This might indeed have been the case in our experiments, keeping in mind the technical limitations of the applied procedures. First, competitively blocking the SRs with poly(I) or DS may have been reversible and incomplete. Second, a knockdown (established using dsRNA injections in the desert locust) does not generate loss-offunction effects to the same degree as a complete knockout; therefore, to date, it remains uncertain whether additional parallel cell entry routes are also functional in the desert locust. 
A
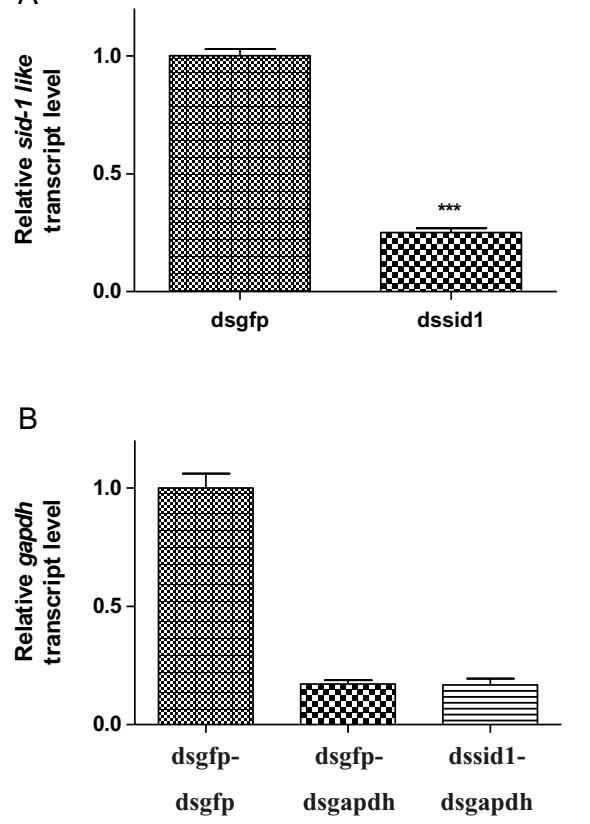

C

sg-SID1

ce-ChUP1

ce-SID1

sg-SID1

ce-ChUP1

ce-SID1

sg-SID1

ce-ChUP1

ce-SID1

sg-SID1

ce-ChUp1

ce-SID1

Sg-SID1

ce-ChUPI

ce-SID1

sg-SID1

ce-ChUP1

ce-SID1

sg-SID1

ce-ChUP 1

ce-SID1
MMGRACCLVLSCVLVHLVQLLHGTEQQTEN--KNLSTI I LNGNYSDYYTFNVNRT-VE- -MRTSQA-- - - - - IFILIFLDSV--- - - - RNQSPQVI PAKWDVVYEKETGHNMSL-- - - - - - MIRVYLI ILMHLVIGLTQNNSTTPSPIITSSNS-SVLVFEISSKMKMIE

- - - - -YIFOFPASEDMIDKPA----RITVECKDSNRSF PVLVV-VROQKGVLSWOLP 作 KKLEANTVHVLRLELDQSFILDLTKVAAEIVDSSKYSKEDGVILEVTVSNGRDSFLLKLP

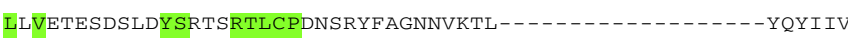
LIVD----NYEYSQVARTLCPFTEY-KEGEAFTV-

............ TVYPNLKLYTDGKLLNPLVEODFGAHRKRHRIGDPHFHONLIV

SISTASNQNLSVSLLVS--- -RKEDFRIRLSDTRNITVL-- - - PAEPQYFAFRFPQSGP - EVTSSRPVHYNFRAE----LVONFYLYNNSORLVTAS-- - - ASEPVYLRYDIPGD-V TVQSRLNADIDYRLHVTHLDRAQYDFLKFKTGQTTKTLSNQKLTFVKPIGFFLNCSEQNI

DTVLLRVDSDSQVCMMVSIQNMSCPVFDLE---QNVHFEGFWETVNVRGGITLP--RDAF DSVAVHLDSNSTICMTVSVQKIGCPVFDLP---DNVNSMGLHQTMTTSATIPVE- -KSRSQFHVTLYSEDDICANLITVPANES IYDRSVISDKTHNRRV-LSFTKRADIFFTETEISM

PLGFYVVFVVKGDDVDCSGPD-----HPIIKP-RNKTLSFSLSPSITYQKYLV----AAC MSSFYVVFVVNTNDDLCSEILSIKPNKPTKF PLRMKSFNVTIESSMKIFDYTIPIVFWAC FKSFRIFVFIAPDDSGCSTNTSRKSFNEKK RIP

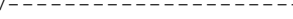

ILLLVTIVVFVYHYFDGIWERR

Figure 5. (A) Silencing of sid1 like was verified in the midgut using quantitative PCR (qPCR), 6 days after injection of $150 \mathrm{ng}$ sid1 like dsRNA ( $\geq 8$, ***: $P<0.0001$ ). (B) Locusts were injected with $150 \mathrm{ng} g f p$ or sid1 like dsRNA and subsequently with $150 \mathrm{ng}$ dsgfp or dsgapdh, indicated as dsgfp-dsgfp, dsgfp-dsgapdh or dssid1-dsgapdh, respectively. The gapdh knockdown was determined $16 \mathrm{~h}$ later with qPCR $(\mathrm{n} \geq 9)$. (C) Sequence comparison of the $\mathrm{N}$-terminal extracellular domain of Sg-SID1 like, Ce-ChUP1 and Ce-SID1 using CLUSTAL OMEGA software. Regions with high similarity between $\mathrm{Ce}$-ChUP1 and Sg-SID1 like are underlined in blue. Poly(I), polyinosine; poly(A), polyadenosine; DS, dextran sulphate; CS, chondroitin sulphate. [Correction added on 10 March, after first online publication: Figure 5(B) was replaced to reflect the correct graph.]

Besides the ability of nematode cells to efficiently take up dsRNA from their extracellular environment via SID1, studies have shown that the RNAi signal is also amplified by RNA-dependent-RNA-polymerases (RdRPs) in these cells (Smardon et al., 2000; Sijen et al., 2001), ensuring high potency and prolonged effectiveness of RNAi. Whereas ticks also possess RdRPs, they seem to have lost sid1-related sequences (Kurscheid et al., 2009). Aung et al. (2011) demonstrated that the hard tick, Haemaphysalis longicornis, uses SRs to mediate envRNAi. Yet, with regard to the importance of RdRPs in systemic gene silencing in plants, fungi and nematodes (Cogoni \& Macino, 1999; Mourrain et al., 2000; Sijen et al., 2001), RdRPs may also attribute to systemic RNAi in ticks. In contrast, knowing that insects lack RdRP-like sequences in their genomes (at least for these insects, whose genomes have been sequenced), it might seem surprising that insects can display robust envRNAi responses. An explanation for the reported high sensitivity of the RNAi response in S. gregaria (Wynant et al., 2012), T. castaneum (Tomoyasu \& Denell, 2004) and L. migratoria (Luo et al., 2012) might reside in the presence of a highly robust cell-autonomous RNAi machinery or an alternative (unidentified) RdRP-independent dsRNA-amplification mechanism. In humans, dsRNA entry is also facilitated by SRs, while it was suggested that long dsRNA induces interferon responses, rather than being an RNAi signal (Limmon et al., 2008; Dieudonne et al., 2012). Nevertheless, this suggests conservation of SR-mediated dsRNA-uptake. Furthermore, several studies have highlighted the importance of endocytosisbased spreading of the RNAi-signal in the nematode, C. elegans (Hinas et al., 2012; Jose et al., 2012).

Direct participation of SR-mediated endocytosis in dsRNA uptake in the desert locust remains to be demonstrated. Yet, since SR-mediated endocytosis is a welldescribed uptake route for polyanionic ligands (Taylor et al., 2005; Pluddemann et al., 2007) and dsRNA uptake via this uptake mechanism has been directly demonstrated in mammalian cell lines (to activate the interferon response) (Limmon et al., 2008; Dieudonne et al., 2012) and in Drosophila S2 cells (Saleh et al., 2006; Ulvila et al., 2006), a role in in vivo dsRNA uptake in S. gregaria might seem plausible.

The use of the RNAi technology as a research tool and as an alternative insect control method is currently hindered because of the poor sensitivity towards envRNAi of many (economically important) insect species, such as D. melanogaster, Bombyx mori, Manduca sexta and Spodoptera litura (Miller et al., 2008; Iga \& Smagghe, 2010; Terenius et al., 2011). Moreover, this has led to the development of (repositories of) RNAi transgenes for 
model organisms, such as D. melanogaster (Dietzl et al., 2007), which is in contrast to the ease of applying RNAi in envRNAi-sensitive insect species, where injection (and in some cases even oral delivery) of dsRNA induces gene silencing throughout the body (Belles, 2010; Huvenne \& Smagghe, 2010). In this context, the knowledge obtained in the present paper may be employed to improve genesilencing techniques in model organisms with reduced envRNAi sensitivity. For instance, this could be achieved by expression of dsRNA-sensitive SRs in Drosophila cells or tissues that are refractory to import dsRNA. An alternative approach may be found in improving the sensitivity of the cell-autonomous RNAi-response by up-regulation of core RNAi-components.

In conclusion, we showed that SR-mediated endocytosis contributes to the envRNAi-response in the desert locust. As the present study is the first report on an in vivo dsRNA transport mechanism in an envRNAi-sensitive insect species, it contributes to a better understanding of the mechanisms of envRNAi in insects and can possibly support the search for the improvement of envRNAi efficiency in other species.

\section{Experimental procedures}

Rearing of the desert locust, Schistocerca gregaria

Gregarious S. gregaria were reared under crowded conditions with controlled temperature $\left(32 \pm 1^{\circ} \mathrm{C}\right)$, light (13 h photoperiod) and relative humidity (40-60\%). They were fed daily with fresh cabbage and dried oat flakes. Adult locusts were developmentally synchronized by transferring them to a different cage directly after their final moult.

\section{Sequence information}

Transcript sequence information of S. gregaria tubu DHQ851397, gapdh DHQ851387, vha16 KJ135006 and clath KJ135005 was retrieved from the annotated $S$. gregaria expressed sequence tag database (Badisco et al., 2011). To verify the sequences, the DNA fragments were cloned into the $\mathrm{PCR} \otimes 4-T O P O \AA$ vector by means of the TOPO TA Cloning ${ }^{\circ}$ Kit for Sequencing (Life Technologies Co, Rockville, MD, USA). The sequences of the inserted DNA fragments were determined using the ABI PRISM BigDye Terminator Ready Reaction Cycle Sequencing Kit (Applied Biosystems, Foster City, CA, USA), while the identity of the tubu and gapdh transcript sequences were previously confirmed by Wynant et al. (2012). The vha16 and clath sequences were compared with BLAST to National Center for Biotechnology Information (NCBI) nucleotide databases of other insects and in silico translated into the corresponding amino acid sequences. The latter were then compared against orthologous sequences of other insects (CLUSTALW) and the predicted protein domains were identified using $\mathrm{CD}$-search (NCBI).

\section{Rapid amplification of $c D N A$ ends}

Only part of the sid1 like transcript sequence of $S$. gregaria was available (Wynant et al., 2012). In order to obtain the complete
ORF cDNA sequence KJ135007, a RACE protocol was performed, following the instructions of the SMARTer ${ }^{T M}$ RACE cDNA Amplification kit (Clontech Laboratories Co., Mountain View, CA, USA). Adapter primers were included in the kit, while genespecific primers were derived from the original PCR fragment: $5^{\prime}$ RACE: CACCAGCCATGCCAATCAAGATGAC; nested 5' RACE: AATAAGCACTAGCATTTATGTCTGGGTG; 3' RACE: TGGCTGG TGTGCTAAAAGGCGGAGTG; nested 3' RACE: TTTGATGTC CGTCCGTGCCAGTCCACTGC. The amplified fragments were analysed with $1 \%$ agarose gel electrophoresis and purified using the GenElute gel extraction kit (Sigma-Aldrich Co., St Louis, MO, USA). The fragments were subsequently cloned in a pCR®4TOPO $\circledast$ vector using the TOPO TA Cloning ${ }^{\circledR}$ Kit for Sequencing (Life Technologies Co.) and sequenced according to the $A B I$ PRISM BigDye Terminator Ready Reaction Cycle Sequencing Kit (Applied Biosystems).

\section{Synthesis of dsRNA}

Double stranded RNAs for clath (561 bp), vha16 (453 bp), sid1 like (250 bp), tubu (545 bp), gapdh (447 bp) and gfp (589 bp) were synthesized using the MEGAscript RNAi kit (Ambion, Austin, TX, USA). A DNA template flanked by two T7 promoter sequences was synthesized for production of clath, vha16, sid1 like, tubu and gapdh dsRNA; therefore, a PCR reaction was performed with cDNA of adult $S$. gregaria midgut tissue, genespecific primers containing a T7 promoter sequence at the $5^{\prime}$ end (Sigma-Aldrich Co.), and REDTaq mix (Sigma-Aldrich Co.) as a source of DNA Taq polymerase, dNTPs and PCR buffer. All PCR primers are shown in the supplementary data (Fig. S3). The amplification products were subsequently analysed by $1 \%$ agarose gel electrophoresis and then visualized with UV light. The PCR product was used directly as template for production of dsRNA. Synthesis of gfp dsRNA was performed using a TOPO 4.1 sequencing vector (Life Technologies Co.) containing a gfp transcript sequence as DNA template, as described in Wynant et al., 2012. After the production of dsRNA, the remaining DNA and ssRNA was removed by nuclease treatment, and proteins and mono/oligonucleotides were removed by solid-phase adsorption purification, according to the manufacturers' specifications (Ambion). The dsRNA-concentration was determined by means of a Nanodrop spectrophotometer (Thermo Fisher Scientific, Inc., Waltham, MA, USA), and the integrity of the dsRNA was assessed by gel electrophoresis using a $1 \%$ agarose gel. The dsRNA was stored at $-20^{\circ} \mathrm{C}$ until further use.

\section{Injection and tissue collection of adult locusts}

Locusts were each injected with $10 \mu \mathrm{l}$ of dsclath, dsvha16, dssid1 like, dstubu, dsgapdh or dsgfp or with poly(I) (Sigma-Aldrich Co.), $\operatorname{poly}(\mathrm{A})$ (Sigma-Aldrich Co.), DS (Sigma-Aldrich Co.) or CS (Sigma-Aldrich Co.) containing solution that was diluted in S. gregaria Ringer solution (1 L: $8.766 \mathrm{~g} \mathrm{NaCl}^{2} 0.188 \mathrm{~g} \mathrm{CaCl}_{2}$; $0.746 \mathrm{~g} \mathrm{KCl} ; 0.407 \mathrm{~g} \mathrm{MgCl}_{2} ; 0.336 \mathrm{~g} \mathrm{NaHCO}_{3} ; 30.807 \mathrm{~g}$ sucrose; $1.892 \mathrm{~g}$ trehalose; $\mathrm{pH} 7.2$ ). Midgut tissue was collected and cleaned by microdissection in $S$. gregaria Ringer solution under a binocular microscope, and immediately transferred to liquid nitrogen to prevent RNA degradation. Samples were stored at $-80^{\circ} \mathrm{C}$ until further processing. 


\section{RNA extraction and cDNA synthesis}

The Lipid tissue extraction kit (Qiagen, Valencia, CA, USA) was used to extract RNA, following the manufacturer's specifications. Quality and concentration of the extracted RNA were assessed using a Nanodrop spectrophotometer. Next, equal amounts of RNA were used as template to produce cDNA. The cDNA synthesis was performed using the PrimeScript ${ }^{\mathrm{TM}}$ First-strand cDNA Synthesis Kit (TaKaRa, Kyoto, Japan), as described in the corresponding protocol. Finally, the cDNA solution was 15-times diluted with MilliQ water (Millapore, Billerica, MA, USA). The resulting cDNA-samples were analysed immediately or stored at $-20{ }^{\circ} \mathrm{C}$ until further usage.

\section{Quantitative real-time PCR}

Primer express software (Applied Biosystems) was used to design qPCR primers. The corresponding primer sequences are shown as supplementary data in Fig. S4. The primers were validated, the most stable reference genes were selected and the qPCR reaction was performed as described in Wynant et al. (2012). To correct for the sample-to-sample variation, the relative transcript levels were normalized against ubiquitin conjugating enzyme 10 and elongation factor 1a. The data were further normalized against a calibrator cDNA sample to account for variation in the PCR efficiency in different PCR runs. In every experiment, no-template controls were included to check for contamination. The PCR reaction was performed and analysed in a 96-well plate and by the StepOne System ( $\mathrm{ABI}$ prism, Applied Biosystems). Since the efficiency of the different primers was the same, the relative transcript quantity was calculated according to the delta-delta Ct method. All data were statistically analysed using non-parametric statistics in GRAPHPAD PRISM 5 (GraphPad).

\section{Acknowledgements}

The authors thank J. Van Duppen, B. Asmelash and F. Van Lommel for technical assistance, R. Jonckers for taking care of the locust cultures and all laboratory staff who took care of the locust cultures on holidays and in the weekends. In addition, the authors gratefully acknowledge the Research Foundation of Flanders (FWO-Flanders), the K.U. Leuven Research Foundation (GOA/11/02) and the Interuniversity Attraction Poles program (Belgian Science Policy Grant P7/40) for financial support.

\section{References}

Abrams, J.M., Lux, A., Steller, H. and Krieger, M. (1992) Macrophages in Drosophila embryos and L2 cells exhibit scavenger receptor-mediated endocytosis. Proc Natl Acad Sci U S A 89: 10375-10379.

Aronstein, K., Pankiw, T. and Saldivar, E. (2006) SID-I is implicated in systemic gene silencing in the honey bee. J Agric Res 45: 20-24.

Aung, K.M., Boldbaatar, D., Umemiya-Shirafuji, R., Liao, M., Xuan, X.N., Suzuki, H. et al. (2011) Scavenger receptor mediates systemic RNA interference in ticks. PLOS ONE 6: e28407.
Badisco, L., Huybrechts, J., Simonet, G., Verlinden, H., Marchal, E., Huybrechts, R. et al. (2011) Transcriptome analysis of the desert locust central nervous system: production and annotation of a Schistocerca gregaria EST database. PLOS ONE 6: e17274.

Baum, J.A., Bogaert, T., Clinton, W., Heck, G.R., Feldmann, P., llagan, O. et al. (2007) Control of coleopteran insect pests through RNA interference. Nat Biotechnol 25: 1322-1326.

Belles, X. (2010) Beyond Drosophila: RNAi in vivo and functional genomics in insects. Annu Rev Entomol 55: 111-128.

Bucher, G., Scholten, J. and Klingler, M. (2002) Parental RNAi in Tribolium (Coleoptera). Curr Biol 12: R85-R86.

Cogoni, C. and Macino, G. (1999) Gene silencing in Neurospora crassa requires a protein homologous to RNA-dependent RNA polymerase. Nature 399: 166-169.

Dietzl, G., Chen, D., Schnorrer, F., Su, K.C., Barinova, Y., Fellner, M. et al. (2007) A genome-wide transgenic RNAi library for conditional gene inactivation in Drosophila. Nature 448: 151U151.

Dieudonne, A., Torres, D., Blanchard, S., Taront, S., Jeannin, P., Delneste, Y. et al. (2012) Scavenger receptors in human airway epithelial cells: role in response to double-stranded RNA. PLOS ONE 7: e41952.

Duxbury, M.S., Ashley, S.W. and Whang, E.E. (2005) RNA interference: a mammalian SID-1 homologue enhances siRNA uptake and gene silencing efficacy in human cells. Biochem Biophys Res Commun 331: 459-463.

Elhassan, M.O., Christie, J. and Duxbury, M.S. (2012) Homo sapiens systemic RNA interference-defective-1 transmembrane family member 1 (SIDT1) protein mediates contact-dependent small RNA transfer and microRNA-21driven chemoresistance. J Biol Chem 287: 5267-5277.

Feinberg, E.H. and Hunter, C.P. (2003) Transport of dsRNA into cells by the transmembrane protein SID-1. Science 301: 1545-1547.

Fire, A., Xu, S., Montgomery, M.K., Kostas, S.A., Driver, S.E. and Mello, C.C. (1998) Potent and specific genetic interference by double-stranded RNA in Caenorhabditis elegans. Nature 391 : 806-811.

Garbutt, J.S. and Reynolds, S.E. (2012) Induction of RNA interference genes by double-stranded RNA; implications for susceptibility to RNA interference. Insect Biochem Mol Biol 42: 621-628.

Hardy, S., Legagneux, V., Audic, Y. and Paillard, L. (2010) Reverse genetics in eukaryotes. Biol Cell 102: 561-580.

Hinas, A., Wright, A.J. and Hunter, C.P. (2012) SID-5 is an endosome-associated protein required for efficient systemic RNAi in C. elegans. Curr Biol 22: 1938-1943.

Hutvagner, G. and Zamore, P.D. (2002) A microRNA in a multipleturnover RNAi enzyme complex. Science 297: 20562060.

Huvenne, H. and Smagghe, G. (2010) Mechanisms of dsRNA uptake in insects and potential of RNAi for pest control: a review. J Insect Physiol 56: 227-235.

Iga, M. and Smagghe, G. (2010) Identification and expression profile of Halloween genes involved in ecdysteroid biosynthesis in Spodoptera littoralis. Peptides 31: 456-467.

Jose, A.M., Kim, Y.A., Leal-Ekman, S. and Hunter, C.P. (2012) Conserved tyrosine kinase promotes the import of silencing RNA into Caenorhabditis elegans cells. Proc Natl Acad Sci U S A 109: 14520-14525. 
Krieger, M. and Herz, J. (1994) Structures and functions of multiligand lipoprotein receptors - macrophage scavenger receptors and Ldl Receptor-Related Protein (Lrp). Annu Rev Biochem 63: 601-637.

Kurscheid, S., Lew-Tabor, A.E., Rodriguez Valle, M., Bruyeres, A.G., Doogan, V.J., Munderloh, U.G. et al. (2009) Evidence of a tick RNAi pathway by comparative genomics and reverse genetics screen of targets with known loss-of-function phenotypes in Drosophila. BMC Mol Biol 10: 26.

Lee, R.M. (1961) The Variation of Blood Volume with Age in the Desert Locust (Schistocerca gregaria). J Insect Physiol 6: 36-51.

Limmon, G.V., Arredouani, M., McCann, K.L., Corn Minor, R.A., Kobzik, L. and Imani, F. (2008) Scavenger receptor class-A is a novel cell surface receptor for double-stranded RNA. FASEB J 22: 159-167.

Lippman, Z. and Martienssen, R. (2004) The role of RNA interference in heterochromatic silencing. Nature 431: 364370.

Luo, Y., Wang, X., Yu, D. and Kang, L. (2012) The SID-1 doublestranded RNA transporter is not required for systemic RNAi in the migratory locust. RNA Biol 9: 663-671.

Mao, Y.B., Cai, W.J., Wang, J.W., Hong, G.J., Tao, X.Y., Wang, L.J. et al. (2007) Silencing a cotton bollworm P450 monooxygenase gene by plant-mediated RNAi impairs larval tolerance of gossypol. Nat Biotechnol 25: 1307-1313.

Miller, S.C., Brown, S.J. and Tomoyasu, Y. (2008) Larval RNAi in Drosophila? Dev Genes Evol 218: 505-510.

Miller, S.C., Miyata, K., Brown, S.J. and Tomoyasu, Y. (2012) Dissecting systemic RNA interference in the red flour beetle Tribolium castaneum: parameters affecting the efficiency of RNAi. PLOS ONE 7: e47431.

Mourrain, P., Beclin, C., Elmayan, T., Feuerbach, F., Godon, C., Morel, J.B. et al. (2000) Arabidopsis SGS2 and SGS3 genes are required for posttranscriptional gene silencing and natural virus resistance. Cell 101: 533-542.

Pearson, A., Lux, A. and Krieger, M. (1995) Expression cloning of dSR-Cl, a class $C$ macrophage-specific scavenger receptor from Drosophila melanogaster. Proc Natl Acad Sci U S A 92: 4056-4060.

Pearson, A.M., Rich, A. and Krieger, M. (1993) Polynucleotide binding to macrophage scavenger receptors depends on the formation of base-quartet-stabilized 4-stranded helices. J Biol Chem 268: 3546-3554.

Perrimon, N., Ni, J.Q. and Perkins, L. (2010) In vivo RNAi: today and tomorrow. Cold Spring Harbor Perspect Biol 2: a003640.

Pluddemann, A., Neyen, C. and Gordon, S. (2007) Macrophage scavenger receptors and host-derived ligands. Methods 43: 207-217.

Ren, R., Xu, X., Lin, T., Weng, S., Liang, H., Huang, M. et al. (2011) Cloning, characterization, and biological function analysis of the SidT2 gene from Siniperca chuatsi. Dev Comp Immunol 35: 692-701.

Saleh, M.C., van Rij, R.P., Hekele, A., Gillis, A., Foley, E., O'Farrell, P.H. et al. (2006) The endocytic pathway mediates cell entry of dsRNA to induce RNAi silencing. Nat Cell Biol 8: 793-802.

Sijen, T., Fleenor, J., Simmer, F., Thijssen, K.L., Parrish, S., Timmons, L. et al. (2001) On the role of RNA amplification in dsRNA-triggered gene silencing. Cell 107: 465-476.
Smardon, A., Spoerke, J.M., Stacey, S.C., Klein, M.E., Mackin, N. and Maine, E.M. (2000) EGO-1 is related to RNA-directed RNA polymerase and functions in germ-line development and RNA interference in C. elegans. Curr Biol 10: 169178.

Swevers, L., Liu, J., Huvenne, H. and Smagghe, G. (2011) Search for limiting factors in the RNAi pathway in silkmoth tissues and the $B m 5$ cell line: the RNA-binding proteins R2D2 and Translin. PLoS ONE 6: e20250.

Taylor, P.R., Martinez-Pomares, L., Stacey, M., Lin, H.H., Brown, G.D. and Gordon, S. (2005) Macrophage receptors and immune recognition. Annu Rev Immunol 23: 901-944.

Terenius, O., Papanicolaou, A., Garbutt, J.S., Eleftherianos, I., Huvenne, H., Kanginakudru, S. et al. (2011) RNA interference in Lepidoptera: an overview of successful and unsuccessful studies and implications for experimental design. $J$ Insect Physiol 57: 231-245.

Tomoyasu, Y. and Denell, R.E. (2004) Larval RNAi in Tribolium (Coleoptera) for analyzing adult development. Dev Genes Evol 214: 575-578.

Tomoyasu, Y., Miller, S.C., Tomita, S., Schoppmeier, M., Grossmann, D. and Bucher, G. (2008) Exploring systemic RNA interference in insects: a genome-wide survey for RNAi genes in Tribolium. Genome Biol 9: R10.

Ulvila, J., Parikka, M., Kleino, A., Sormunen, R., Ezekowitz, R.A., Kocks, C. et al. (2006) Double-stranded RNA is internalized by scavenger receptor-mediated endocytosis in Drosophila S2 cells. J Biol Chem 281: 14370-14375.

Valdes, V.J., Athie, A., Salinas, L.S., Navarro, R.E. and Vaca, L. (2012) CUP-1 is a novel protein involved in dietary cholesterol uptake in Caenorhabditis elegans. PLoS ONE 7: e33962.

Waterhouse, P.M., Wang, M.B. and Lough, T. (2001) Gene silencing as an adaptive defence against viruses. Nature 411: 834842.

Whangbo, J.S. and Hunter, C.P. (2008) Environmental RNA interference. Trends Genet 24: 297-305.

Whitman, S.C., Daugherty, A. and Post, S.R. (2000) Regulation of acetylated low density lipoprotein uptake in macrophages by pertussis toxin-sensitive G proteins. J Lipid Res 41: 807813.

Winston, W.M., Molodowitch, C. and Hunter, C.P. (2002) Systemic RNAi in $C$. elegans requires the putative transmembrane protein SID-1. Science 295: 2456-2459.

Wynant, N., Verlinden, H., Breugelmans, B., Simonet, G. and Vanden Broeck, J. (2012) Tissue-dependence and sensitivity of the systemic RNA interference response in the desert locust, Schistocerca gregaria. Insect Biochem Mol Biol 42: 911-917.

Yu, N., Christiaens, O., Liu, J.S., Niu, J.Z., Cappelle, K., Caccia, S. et al. (2013) Delivery of dsRNA for RNAi in insects: an overview and future directions. Insect Science 20: $4-14$.

Zambon, R.A., Vakharia, V.N. and Wu, L.P. (2006) RNAi is an antiviral immune response against a dsRNA virus in Drosophila melanogaster. Cell Microbiol 8: 880-889.

\section{Supporting Information}

Additional Supporting Information may be found in the online version of this article at the publisher's web-site: 
Figure S1. Six days after the injection of sid1 like or gfp dsRNA, the tubu transcript level was determined using quantitative PCR $(P<0.014, n \geq 7)$.

Figure S2. The predicted topological structure of Ce-ChUP1 using PHOBIUs software.
Figure S3. Sequences of the primers used to make the template for dsRNA production. The T7-promotor site is indicated in bold.

Figure S4. Sequences of the quantitative PCR primers. 